\title{
POSITIVE EFFECT OF INCREASING FEEDBACK FOR STUDENT SELF-ADJUSTMENT OF LEARNING HABITS
}

\author{
Ramon Vilanova ${ }^{1}$, Pedro Ponsa $^{2}$ \\ ${ }^{1}$ Dept. Telecommunications and Systems Engineering, Universitat Autònoma de Barcelona. \\ ${ }^{2}$ Dept. Systems, Automatic and Control Engineering, Universitat Politècnica de Catalunya \\ Barcelona, Spain \\ ramon.vilanova@uab.cat
}

\begin{abstract}
Universities are currently undergoing a significant amount of changes of a conceptual and methodological nature, in compliance with the European Higher Education Area (EHEA). This new scenario promotes new learning and teaching methodologies under specific criteria. In a global sense it can be stated that the main outcome of this new approach is the increased level of feedback required amongst the principal actors in question throughout the learning process (e.g. lecturer and students). In this article we present a scheme in which the organization of the lecture activities (teaching, evaluation, assessment, etc) are organized according to a timeline that respects the overall aim of increasing the amount of feedback a given student receives. Although it may be widely recognized that on-going evaluation constitutes a positive framework that can enhance student performance, this study demonstrates that if all the activities are designed as a whole, the self-feedback of the student is increased and the overall performance improved. The teaching of the subject of "Signals and Systems"; a component of the Computer Science Engineering degree, is used as a case study for this experience. It has been used as a framework and the improvements developed with respect to previous years achieved have been demonstrated with data.
\end{abstract}

Keywords - Continuous Evaluation, student self-learning, feedback

\section{INTRODUCTION}

The changes to the university educational system; based on the guidelines of the European Higher Education Area (EHEA), that are currently underway [1] give rise to methodological and conceptual challenges throughout the entire university system. One important aspect is the shift towards continuous evaluation procedures that provide continuous student feedback on competence level acquisition. This new approach thus requires both new information and new work procedures, which in turn lead to the important issue of the handicap of relating resource requirements to the performance of continuous evaluation, and to those resources already in existence. There is also a tendency to promote student self study in that the student should be able to find resources aimed at facilitating the self-study approach.

The approach presented here is embedded within the Instructional Feedback paradigm [2], and takes into account the different actors and how they interact during the learning process. Although it may appear to be a rather evident conceptual framework, in the opinion of the author of this study, this approach is flawed, is commonplace in the academic sector, and doesn't take out the time to reflect on the way we teach and in addition question, many of those actions which are habitually implicit in nature.

Instructional Feedback is based on the basic notion of feedback; now a well established mechanism in engineering applied to the development automatic control systems $[3,4]$. The main benefits of feedback come from the generation of an error signal between a desired target and its observed output. The main actors of a feedback control system are the process or system to be controlled and the controller. The controller acts on the system by generating appropriate control actions according to the observed feedback error (target observed system's output).

The Instructional Feedback model [2] establishes a parallelism between the previously described situation and the relationships between teacher/instructor and student/learner. In such a model the teacher/instructor is 
considered the controller or energy provider of the system in question (the student's/learner's skills and competence in a specific subject). The instructional feedback is a closed loop model that relies on feedback received from the students/learners as well as the generation of different actions designed to enhance their learning performance.

In this article, the idea of instructional feedback is reviewed and slightly reformulated; highlighting the necessity and importance of another feedback loop that ought to be closed on the student/learner side while outlining the importance of providing the means for this to take place. In the conventional instructional feedback model feedback is received on the teacher/instructor side, whereas, in the author's view, it is on the student side where is has to be closed in order to be effective.

This approach was been tested on the teaching of Signals and Systems to Computer Science students of an Engineering degree. Following a revision of the instructional feedback model, at this stage an introduction is provided on how this model is modified and implanted. The method was applied for a two year period while being compared with the student performance of with previous years. According to the author, this alternate proposal for the instructional feedback model constitutes, an alternative way of looking at continuous evaluation [5], in which the main goal is not to merely asses the student/learner as they advance through the syllabus, but also to provide a means for self-study and motivation [6] in the subjects that require most attention.

The main contribution of this paper is the instruction, based on real experience, on how the development of appropriate feedback channels can lead to improved academic results. The applied scheme is explained in detail as comparisons with the continuous evaluation method are also discussed. Academic results after two years of execution indicate that this approach essentially decreases the number of no-shows and demonstrates slightly higher percentiles of students in the higher mark range of the class.

The rest of the paper is organized as follows; Section 2 is devoted to the presentation of the different elements as well as the framework used by the paper (namely the instructional feedback scenario and a brief review of a feedback based control system), Section 3 places continuous evaluation within the previously addressed instructional feedback; highlighting (from the student's perspective) the necessity for generating more comprehensive feedback information. Section 4 presents the Signals and System's subject in which the proposal was implemented (content as well as performance indicators for a total of three academic years are explained). Section 5 provides a detailed exposition of the different activities proposed, their distribution throughout the semester as well as the rationale for their precise time organization. The paper concludes with a summary of the content and a discussion of some of the main conclusions.

\section{MATERIALS AND METHODS}

This section will present the basic conceptual idea of a feedback control loop and its extension within the educational framework, by means of an Instructional Feedback scenario.

\subsection{Feedback control}

Feedback has recently become a well established mechanism in applied engineering used in the development of automatic control systems $[3,4]$. The main benefits of feedback occur via the generation of an error signal between the desired target and the observed output. The key performers of a feedback control system are the process or system to be controlled and the controller, which acts on the system by generating appropriate control actions based on the observed feedback error (target - observed system's output). As a familiar example, we can consider room temperature control: on the thermostat we set the desired room temperature (i.e. the target temperature), then the temperature is detected (the observed system output) by and transferred to the thermostat that adjusts the heating/ventilating system accordingly in order to achieve the desired target temperature.

Feedback control is a very important theory in scientific applications. An example of this is its extensive use in dynamic systems such as the regulation of temperature and humidity required to make living spaces more comfortable. The nature of this system generally implies a type of physical entity in which an action is executed via an input. The system subsequently responds to this input by producing an output, and furthermore, could be classified as dynamic when the phenomena described occurs in a time-domain. 
Fig. 1 shows a typical feedback control system, which comprises a controller unit and plant unit; with the former affecting the performance of the latter. In other words, the controller output determines the plant output. A controller must be designed to reach the system goal. The controller type can be proportional (P), integral (I), or derivative (D), or a combination of any of the three. The input of the whole system is called a reference value, the controller output a manipulated variable, and the plant output is termed a controlled variable.

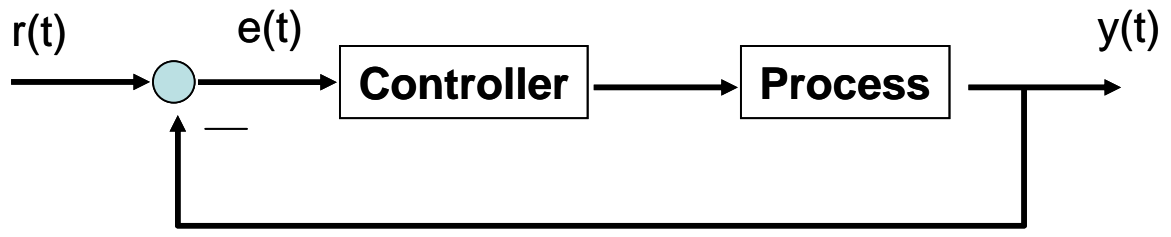

Fig. 1: Feedback Control system. $r(t)$ corresponds to the desired or reference value for the process output $y(t)$, while $e(t)$ is the error signal computed as $e(t)=r(t)-y(t)$.

\subsection{Instructional Feedback}

Instructional feedback (IF) is presented as the application of theoretical control concepts to the teaching/learning domain. The basic diagram showing the different actors is shown in Fig. 2.

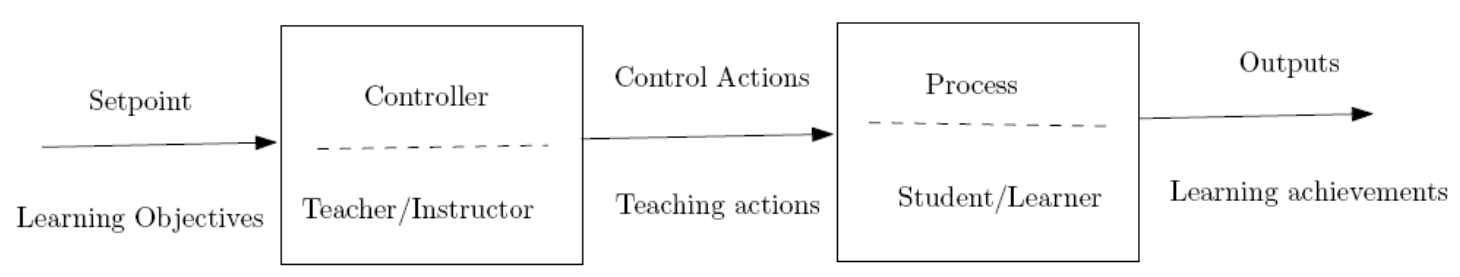

Fig. 2: Open-loop instructional/control system

This figure outlines the main elements of the teacher/learner collaborative system and its counterparts from the process control system domain. As can be observed, this system corresponds to what it is known as an open-loop control system. The primary objective of the control system is the achievement of a set of learning outcomes by the student (the learner). These learning outcomes enter the teacher/ instructor phase, where an appropriate set of teaching actions are generated that will in turn drive the learner towards the attainment of more of such learning outcomes.

As it has been mentioned, the control systems theory states that this scheme corresponds to an open-loop system, meaning that the instructor generates teaching actions based on the learning outcomes and not on the actual achievement of the learning outcomes by the learner. Therefore, the teaching actions are a pre-defined set of actions that are conceived a priori and are independent of the behaviour exhibited by the controlled process (in this case, the learner). The open-loop approach for controlling a system is recognized, for several motives, as an inefficient way of controlling a system $[3,4]$.

The drawbacks of the open-loop approach to control give rise to a more efficient approach: one based on closed-loop feedback control. In this new scheme the controller makes decisions based on a continuous monitoring of the process outputs, meaning that new control actions are generated in the case that the previous ones should not be efficient enough. At this point the decision as to whether or not the loop should be closed takes us back to the feedback-based Instructional Learning system shown in Fig. 3. 


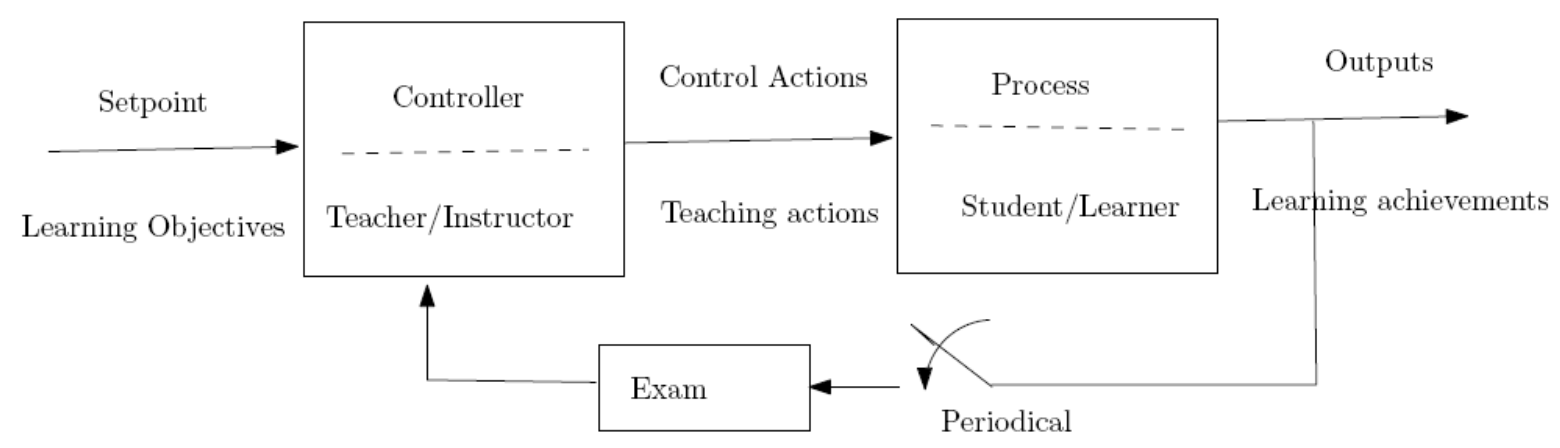

Fig. 3: Closed-loop instructional/control system

As is habitual, the controller is informed via a measurement of the actual output of the process. It has to be noted, however, that the feedback mechanism is associated with a sampling time that causes it to operate on a periodical basis. The consequence of this is the fact that the loop is not closed continuously but rather, only at certain precise instants (from an instructional view point, the moments in which evaluations or exams are performed): within the control theory domain, these instants are called sampling instants. Our next task will be to reinterpret selected well known teaching/learning models related to the feedback control system, with the main task being to show that the genuine use of feedback is a rather misunderstood concept.

\section{CONTINUOUS EVALUATION AND INSTRUCTIONAL FEEDBACK}

First of all it has to be made clear that in a feedback control system, the control actions are constructed on the basis of previous history of process output and previous control actions. This fact allows the controller to evaluate whether the output of the process is geared towards the right direction. More formally, let us assume that the time evolves according to a certain time unit, (one day, one hour, one week, etc). In this sense, as $k$ time units we can think of a vector of length $k$ as containing the values of a variable of interest in each one of such time instants. For example, if we denominate the learning outcomes at time instant $k$ as $r_{k}$, the result will be that vector $r_{k}=\left(r_{0}, \ldots . r_{k}\right)$ will contain all previous values. Along the same lines we denote $u_{k}=\left(u_{0}, \ldots . u_{k}\right)$ and $\mathbf{y}_{\mathrm{k}}=\left(\mathrm{y}_{\mathrm{o}}, \ldots . \mathrm{y}_{\mathrm{k}}\right)$ as the control or teaching actions and the learner outputs respectively. Based on these definitions, a feedback-based control action can be written as

$$
u_{k}=f\left(r_{k}, u_{k-1}, y_{k}\right)
$$

Whereas in the case that non-use of information regarding the values of the process output on previous instant is considered, the control action can be written as a function of the form

$$
u_{k}=f\left(r_{k}, u_{k-1}\right)
$$

In any regard, the sampling action corresponds to the execution of one or more evaluation tasks that will inform the instructor about student progress. In this sense sampling can take on various forms depending on the evaluation performed.

\subsection{End-of-Term Evaluation}

Performing an end-of-term evaluation (EOT) is a classical approach that is currently being replaced by continuous evaluation. Within the EoT approach the students are examined at the end of the teaching period. Students are assessed on the basis of this evaluation, in which even the instructor receives information (acquired at the end of the term) about the student's progress. Therefore, as in all teaching (control) actions, these are generated independently of such progress; i.e. it is an open-loop- based instructional system.

Nevertheless, there is a possibility for closed-loop actions within the EoT framework if we implement a different time scale; on a term-to-term basis for example. At such a scale we can apply feedback by learning as we go from one term to the next. Teaching actions can therefore be updated or modified in order to increase their efficiency, thus leading to a greater likelihood of them achieving the learning outcomes in question. This framework is that which is typically encountered in our faculties. However, the question remains as to whether or not feedback is really applied? In the author's view, this is not the case, as the teaching actions are basically the same (or at least their effectiveness is not assessed) from term to term. 
Furthermore, from a more meticulous control point of view one can observe that, theoretically, from term to term there is a change in the system to be controlled; the make-up of the student population is not consistent. Henceforth, if we were to carry out an additional process, the problem would be different from the previous one, meaning in essence that feedback wouldn't be possible.

\subsection{Continuous Evaluation}

Continuous evaluation (CE) is recognized as an improvement to its EoT counterpart. In this approach, the periodic application of evaluation actions provides the teacher with valuable information about student progress. It must be noted once again that, in order to fully exploit the potential of feedback, a record of all teaching actions must be taken following each evaluation. This explicitly means that the results of an evaluation should be compiled not only to assess the student's progress but also to give the instructor an opportunity to evaluate the effectiveness of the teaching actions applied. This second observation is considered a missing term that the authors of this study feel could help improve teaching quality.

Hence there is a misunderstanding concerning the notion that CE is a means of introducing feedback into the teaching process. Whilst there is a need for generating information; accomplished by the different evaluations periodically performed, there is also a need for such information to be used.

Two potential users of this information have thus been identified and therefore, two feedback loops can be closed.

- Feedback loop from the instructor's/teacher's end: As previously mentioned, this implies the evaluation of the effectiveness of the teaching actions applied and suggests techniques for the improvement of the same.

- Feedback loop from the learner's/student's end: From the periodical results the student should perform a personal evaluation of the way the study is conducted and how the study habits could be changed in order to take full advantage of the educational resources available.

The recognition of these two feedback loops and the way in which the two can be applied is a potential way of improving present day trends in teaching $t$ [7]. The next section outlines how this second feedback loop can be created as well as how a self-assessment can be induced.

\section{FEEDBACK IMPROVEMENT IN SIGNALS AND SYSTEMS}

In this section we present the "Signals and Systems" subject within the same context as mentioned previously. We highlight the major difficulties faced by students and determine the principal challenges that are to be faced in order to ensure improvement.

\subsection{SUBJECT DESCRIPTION}

The subject at hand study is entitled "Signals and Systems (SS)" and is given at the Technical University School of Engineering at the Universitat Autònoma de Barcelona (Barcelona, Spain). "Signals and Systems" is a 5 ECTS subject of the Computer Science Engineering (CSE) degree and is a compulsory requirement for the CSE degree. Currently, SS is given in the forth semester, with the degree consisting of 6 semesters. In order to meet the prerequisites for the subject students must have a satisfactory level of competence in complex analysis and basic calculus.

Teaching is scheduled for a 16 week period in which the student has two distinct types of lecture each week. The first of which is a two-hour theory lecture in which the theoretical contents of the subject are introduced and explained to the student. Secondly there is a one-hour practical lecture in which the theoretic concepts introduced previously are implemented in practical assignments.

Moreover, five two-hour laboratory sessions are set up during the semester in which the students implement computer programs of selected topics.

The evaluation of each subject is as follows: $70 \%$ of the student evaluation comes from the assessment of the theoretical components. The remaining $30 \%$ is evaluated in laboratory sessions. 


\subsection{Content}

The subject content is centered primarily on continuous time signals and systems, and covers the following topics:

1. Introduction (1 week). The objective of this topic is twofold. The first of which is the introduction of the subject content and assessment protocol. The second is to contextualize the SS within the CSE degree and highlight its applications in fields such as image processing, control engineering and communications.

2. Linear Systems (1 week). In this topic the basic concepts of "Signal and Systems" are introduced as well as basic properties and linearity. The concept of discrete SS and periodicity is introduced. Properties of discrete sinusoidal signals are tested and compared with the continuous counterpart.

3. Laplace Transform (3 weeks). One of the main tools in SS, the Laplace transform definition is overviewed and used to solve differential equations. Furthermore Laplace transforms are applied to compute the output of a system.

4. System Analysis ( 2 weeks): First and second order systems are defined and their transfer function computed with respect to the Laplace transform. Main properties and features of their outputs are presented.

5. Fourier Analysis ( 3 weeks): This is presented as the Laplace counterpart for signals and comprises the discrete Fourier series for periodic signals and the Fourier Transform generalization to non-periodic signals. From a practical standpoint, the Fast Fourier Transform is also introduced.

6. Frequency Response ( 1 week): Being the last topic to be covered, this joins the transfer function representation of a system with the frequency domain of a signal by way of the Fourier analysis. Initial ideas on filtering and modulation applications are introduced.

A total of 11 weeks are devoted to lectures. Four (4) intermediate weeks are reserved for evaluation whilst the remaining week is reserved for the scheduling of local holidays.

\subsection{Situation before the application of the proposed methodology}

A background perspective is presented first in order to better evaluate the results presented below. First of all it must be pointed that the three year evaluation shown in Table 1 was carried out based on a two-round system in which the students sat an end-of-term exam. Those who were unsuccessful were granted another opportunity at the end of the following semester without being required to 're-attend' the lectures. For each one of the academic years the following data has been presented:

- Number of students matriculated each academic year.

- The percentage of students evaluated: This is the number of matriculated students that actually take the exam (taking into account the two possible rounds).

- The percentage of students that pass the subject: This value is calculated in relation with the number of matriculated students. We do not consider the percentage with respect to the number of students that take the exam, given that some of them set the exam using both rounds. Hence this is difficult to summarize.

- The percentage of students that pass the subject with an A Grade (best) or C Grade: Note that here grade $A$ means students that score above 8.5 out of 10 , grade B students that score between 7 and 8.5 out of 10 and grade $C$ students that score between 5 and 7 out of total of 10 marks.

Table 1: 'Signals and Systems' subject background data

\begin{tabular}{|l|c|c|c|}
\hline \multicolumn{1}{|c|}{ Academic Year } & $2004 / 2005$ & $2005 / 2006$ & $2006 / 2007$ \\
\hline Matriculated & 174 & 153 & 154 \\
\hline
\end{tabular}




\begin{tabular}{|l|c|c|c|}
\hline No Show/Matriculated & $29 \%$ & $35 \%$ & $37 \%$ \\
\hline Success / Matriculated & $60 \%$ & $54 \%$ & $51 \%$ \\
\hline Grade A / Success & $6 \%$ & $13 \%$ & $15 \%$ \\
\hline Grade B / Success & $29 \%$ & $29 \%$ & $28 \%$ \\
\hline Grade C / Success & $66 \%$ & $57 \%$ & $57 \%$ \\
\hline
\end{tabular}

Several points are to be noted from the data on Table 1:

- First of all, one can observe that after performing the two rounds, the number of students that do not take the examination is always over $30 \%$. Hence these students neither follow the subject nor do they take any exam (in any of the two rounds).

- The number of students that succeed in passing this subject is approximately $50 \%$ of the total number of those matriculated.

- Most of the successful students (approximately 60\%) attained a $C$ grade.

These conclusions underline the fact that students found the subject matter rather difficult to follow and encountered difficulties in acquiring the knowledge required at that particular level. This has the negative side effect of not equipping students satisfactorily for the subjects to follow, nor for those additional subjects which demand a background understanding of the subject matter at hand. In most cases, even those that do in fact succeed in passing 'Signals and Systems' are aware that the subject contents are not dealt with adequately. Measures therefore need to be put in place which can improve these factors.

The contents of 'Signals and Systems' are rather mathematical in nature and students are encouraged to practice with mathematical problems. In the light of this reality, each week there is a one-hour session in which problems associated with the theoretical components are formulated. Students are even given the problems in advance as a means of encouraging them to put them to practice; understanding that students normally are used to waiting until they reach the classroom before they concern themselves about solving such problems.

\subsection{Subject Activities Organization and Feedback Loop Identification}

Activities can be categorized as; 1 ) theoretical front-teaching lectures; 2 ) problems sessions in which problems are solved and 3) computer lab sessions. We refer to these as theoretical, problem and practical sessions respectively. The distribution of these activities during the 16 weeks of the term is as follows in Table 2:

Table 2: Weekly distribution of subject activities and duration in hours

\begin{tabular}{|c|c|c|c|c|c|c|c|c|}
\hline Week & $\bar{T}$ & $\mathbf{S}$ & PS & $\mathbf{L}$ & $\overline{\mathrm{PL}}$ & $\mathbf{S}$ & EA & Total \\
\hline \begin{tabular}{l|l}
$122-$ set/26-set \\
\end{tabular} & 1,5 & & & & & 3 & & 4,5 \\
\hline \begin{tabular}{l|l}
2 & $29-$ set/3-oct
\end{tabular} & 1,5 & & 2 & & & 3 & & 6,5 \\
\hline \begin{tabular}{l|l}
3 & 6 -oct/10-oct
\end{tabular} & 1,5 & 1 & 2 & 2 & 1 & 3 & & 10,5 \\
\hline \begin{tabular}{l|l}
4 & $13-o c t / 17-o c t$ \\
\end{tabular} & 1,5 & 1 & 2 & & & 3 & & 7,5 \\
\hline \begin{tabular}{l|l|}
5 & 20 -oct/24-oct \\
\end{tabular} & 1,5 & 1 & 2 & & & 3 & & 7,5 \\
\hline \begin{tabular}{l|l}
6 & $27-o c t / 31-o c t$ \\
\end{tabular} & & 1 & & 2 & 1 & 4 & 1 & 9 \\
\hline \begin{tabular}{l|l}
7 & $3-n o v / 7-n o v$
\end{tabular} & 1,5 & 1 & 2 & & & 3 & & 7,5 \\
\hline \begin{tabular}{l|l}
8 & $10-n o v / 14-n o v$ \\
\end{tabular} & 1,5 & 1 & 2 & & & 3 & & 7,5 \\
\hline \begin{tabular}{l|l}
9 & $17-n o v / 21-n o v$ \\
\end{tabular} & & 1 & 1 & 2 & 1 & 6 & 1 & 11 \\
\hline \begin{tabular}{l|l}
10 & $24-n o v / 28-n o v$ \\
\end{tabular} & 1,5 & 1 & 2 & & & 3 & & 7,5 \\
\hline \begin{tabular}{l|l}
11 & $1-d e s / 5-d e s$ \\
\end{tabular} & 1,5 & 1 & 2 & & & 3 & & 7,5 \\
\hline \begin{tabular}{l|l}
12 & $8-d e s / 12-d e s$ \\
\end{tabular} & 1,5 & & 2 & & & 3 & & 6,5 \\
\hline \begin{tabular}{l|l}
13 & $15-d e s / 19-d e s$
\end{tabular} & & 1 & & 2 & 1 & 6 & 1 & 11 \\
\hline \begin{tabular}{l|l|}
14 & $22-$ des$/ 9-g e n$ \\
\end{tabular} & 1,5 & 1 & 2 & & & 3 & & 7,5 \\
\hline \begin{tabular}{l|l}
15 & $12-g e n / 16-g e n$ \\
\end{tabular} & & 1 & & 2 & 1 & 6 & 1 & 11 \\
\hline \begin{tabular}{l|l}
16 & $19-g e n / 23-g e n$
\end{tabular} & 1,5 & 1 & & & & & & 2,5 \\
\hline Total & 18 & 13 & 20 & 10 & 5 & 55 & 4 & 125 \\
\hline$\%$ & $14,40 \%$ & $10,40 \%$ & $16,00 \%$ & $8,00 \%$ & $4,00 \%$ & $44,00 \%$ & $3,20 \%$ & $100,00 \%$ \\
\hline
\end{tabular}


Where T stands for Theory; S for seminars (problems and exercises); PS for Preparation for Seminars; $L$ for Computer Lab sessions; PL for preparation for Lab; S for the student's own study hours and EA for Evaluation activities. The subject has a total of 5 ECTS (125 dedication hours).

What is more important than the weekly distribution of activities within each thematic, is their organization along the term timeline. Two different kinds of sequencing are programmed:

i.) The Theory $\rightarrow$ Problems $\rightarrow$ Practical sequencing; providing an appropriate framework with which the concepts presented could be better established.

ii.) The Evaluation $\rightarrow$ Assessment sequencing where the student is periodically evaluated.

If we consider a time arrow representation of the previous sequencings we have the following figure, Fig. 4:

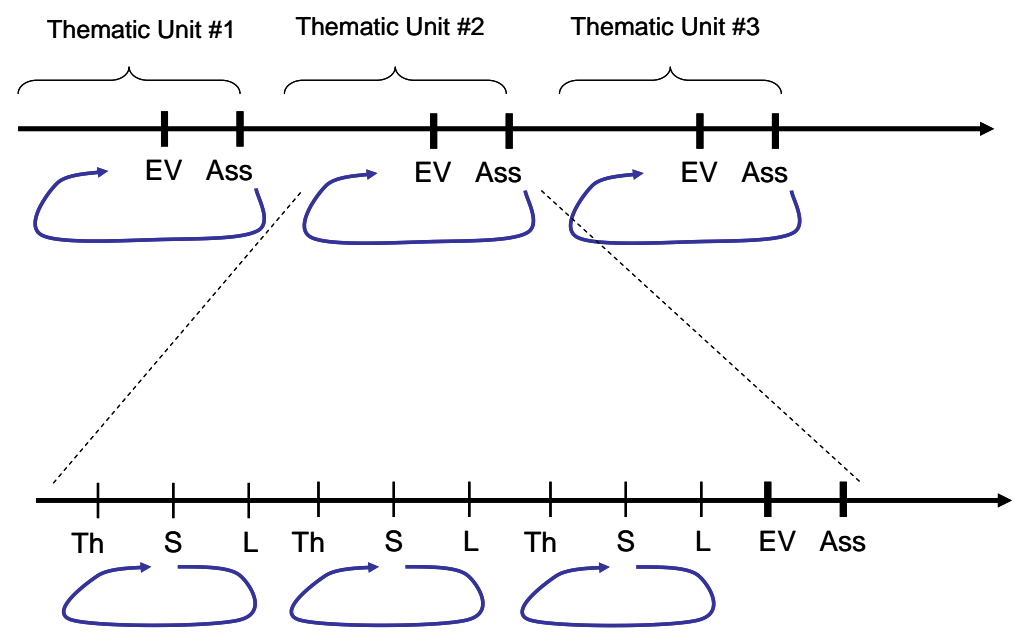

Fig. 4: Timeline perspective of subject activities organization. For each thematic unit there is a sequence of feedback loops defined according to the teaching activities.

Thanks to the sequencing outlined in point ' $i$ ', we achieve a somewhat typical sequencing that permits an initial feedback level that is executed on a weekly basis (after presenting the concepts from a theoretical point of view, the student can complement the activity by practicing with problems and computer lab sessions). Considerable effort has gone into the pursuit of these three sessions in the correct time sequencing. Indeed, most subjects do have these three kinds of teaching sessions. Computer lab sessions, for example, are sometimes performed just after (or before for that matter!) the presentation of the corresponding theoretical concepts. In this manner, appropriate sequencing provides the means for imparting the concepts and helping to identify any points that should warrant more attention.

Once each thematic unit has been completed, an evaluation session is conducted by using the time slot allocated to a theoretical session (therefore not introducing any new concepts till the previous thematic unit has been completed and evaluated). In this manner, the next three time slots which are associated with a theory session, problem session and computer lab session are instead employed for the following use described below and continued to the next unit the following week:

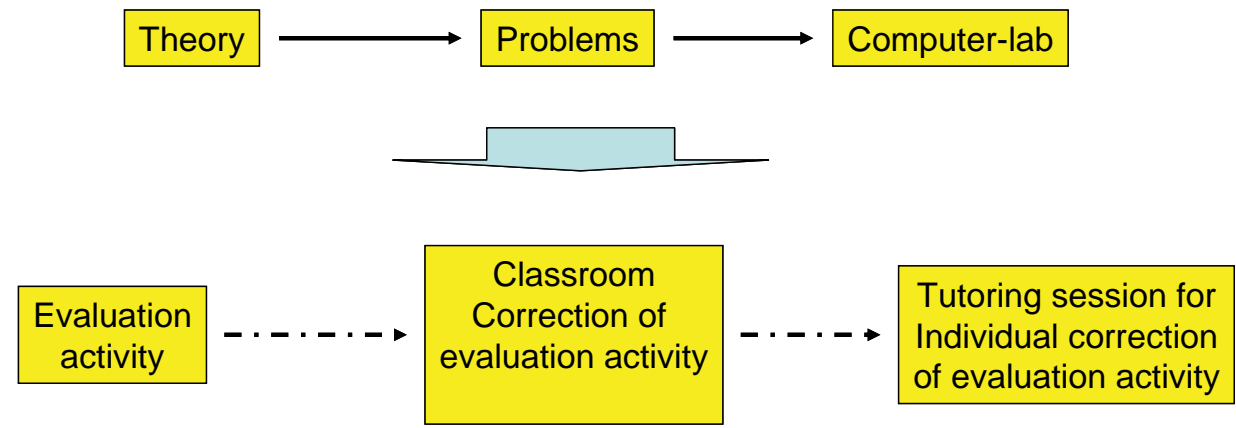

Fig. 5: Activities reassignment for the evaluation/assessment week 
As a result, the week corresponding to these three time slots lays the foundation for the second level of feedback (associated to the thematic unit as a whole). At this point the student will receive some help for the generation of such corrective actions:

i.) If the student is successful in the evaluation activity they are encouraged to continue along the same lines or they may even be suggested to go into further detail in certain concepts. The objective of this is to introduce them to the field of research.

ii.) The student who, despite having met the criteria, has not scored satisfactorily on the assessment: If there are signs that the student can clearly perform better, those aspects in which the student failed are identified and corrective actions are proposed. The student will have another opportunity (at the end of the term) to evaluate and improve their overall score.

iii.) The student fails to succeed:

As with point 'ii', points of failure are identified and corrective actions are delimited.

This second feedback loop allows the student to assess how the thematic unit has been tackled. The benefits of such an assessment will be twofold: firstly, the student can determine to what extent time dedication and the study methods used proved to be most useful. Regarding the specific thematic unit, the student should evaluate if the specific contents prove to be too difficult. For these reasons, more time should be devoted to its study.

On the contrary, the information provided in this assessment can give the student the opportunity to reconsider the use of more effective learning methodologies for subsequent thematic units.

This second feedback loop, applied to each unit, allows the student to perform a self assessment and also better prepare themselves (if necessary) for the final subject evaluation. In fact, at the end of the term the student will be allowed a second attempt at each one of the units. In essence, the aim of these teaching and evaluation activities is to go one step beyond continuous evaluation. Indeed, between two consecutive thematic units there is an evaluation/assessment week during which no new content is introduced and is devoted entirely to the generation of the necessary feedback information.

Continuous evaluation typically comprises an evaluation activity that is performed in which the student obtains a corresponding score. Here, two different levels of correction and assessment of the solutions are performed; one at the classroom level (so the student will know how he or she performs with respect to their classmates) and a second one at personal level.

The third and most important feedback period takes place at the end of the term. Evidently, the main issue here is that this feedback information is not useful for improving the subject performance during the semester underway at the time. However, the student is able to draw certain conclusions and, as they will have already undergone a pre- evaluation in each thematic field, the outcome of any relevant corrective actions applied could be evaluated. The drawing of conclusions will therefore be beneficial, even in the case that the student should fail to pass the subject (lessons learned will be put to use in subsequent areas). Should the student be successful, it would enable them to be better prepared for the subjects to come.

\subsection{Facts and Figures after two years}

After two years of running this subject, coupled with the introduction of the activity organization as presented in the previous section, statistics changed only slightly with respect to the ones presented above. These results are showed at Table 3.

Table 3: Figures for the last two years

\begin{tabular}{|l|c|c|}
\hline \multicolumn{1}{|c|}{ Academic Year } & $2007 / 2008$ & $2008 / 2009$ \\
\hline Matriculated & 136 & 102 \\
\hline Non Show/Matriculated & $21 \%$ & $25 \%$ \\
\hline Success / Matriculated & $68 \%$ & $74 \%$ \\
\hline
\end{tabular}




\begin{tabular}{|l|c|c|}
\hline Grade A / Success & $3 \%$ & $15 \%$ \\
\hline Grade B / Success & $48 \%$ & $81 \%$ \\
\hline Grade C / Success & $49 \%$ & $39 \%$ \\
\hline
\end{tabular}

As it can be seen, the percentage of students that succeed in passing the subject is shown to have increased notably. Be that as it may, a few additional years will be required in order to observe any clear trends. Nevertheless, an important outcome can be observed regarding the grades achieved by the students that do pass the subject; the majority of the students achieve a $B$ grade as opposed to passing with a mere $C$ grade as had been the case previously. This thus indicates an acquisition of an enhanced level of knowledge of the subject contents. It can be concluded therefore that there are benefits on both sides.

\section{CONCLUSIONS}

In this paper the impact of activity organization on student performance is demonstrated. More specifically, the organization provided is oriented towards the generation of self-assessment opportunities. The discussion has been centred on the instructional feedback model as an appropriate framework for identifying the feedback possibilities within a teacher-learner context.

Concerning the need for generating effective feedback information from the student's end, at the student side, an organization of teaching and evaluation activities has been presented. These activities have been applied for the last two academic years in the "Signals and Systems" subject.

The results presented indicate that there is an improvement in the number of students who pass the subject and, more importantly, the ones that pass the subject obtain better grades. It is therefore considered that if new activities (or the re-design of current ones) are carried out bearing in mind the fact that the generation of feedback information for the students, the cost/benefit ratio in terms of student satisfaction and consolidation of concepts presented will increase.

\section{ACKNOWLEDGEMENTS}

The authors would like to express our gratitude for the financial support granted by the Universitat Autonoma de Barcelona and Universitat Politècnica de Catalunya under their respective MQD (improvement in teaching quality) programs.

\section{REFERENCES}

1. The Bologna Process - Towards the European Higher Education Area, http://ec.europa.eu/education/policies/educ/bologna/bologna_en.html, Accessed 19 October 2010

2. Darrell L. Vines and James R. Rowland, An Instructional Feedback Model for Improved Learning and Mentoring, IEEE FIE - Frontiers in Education Conference, 1995

3. G. F. Franklin; J. D. Powell and A. Emami-Naeini, Feedback Control of Dynamic Systems, Prentice-Hall, 2009-2010

4. Paul H. Lewis and Chang Yang, Control Systems in Engineering, Prentice-Hall, 1999

5. C. Coll; M.J. Rochera; R.M. Mayordomo and M. Naranjo, Continuous assessment and support for learning: an experience in educational innovation with ICT support in Higher education, Electronic Journal of Research in Educational Psychology, Vol 5 (3), ISSN 1696-295, December 2007, pp 783-804

6. A. Rizwan; M.S.I. Alvi and M.M.I. Hammouda, Analysis of Factors Affecting the Satisfaction Level of Engineering Students, International Journal of Engineering Education, Vol 24, (4), 2008, pp 811-816 
7. P.Ponsa, B. Amante, J.A. Roman, S. Oliver, M. Diaz, J. Vives, Higher Education Challenges: Introduction of Active Methodologies in Engineering Curricula. International Journal of Engineering Education, Vol 25, (4), 2009, pp 799-813 